\title{
INFECCION POR CHLAMYDIA TRACHOMATIS, CANDIDA ALBICANS $Y$ NEISSERIA GONORRHOEAE EN MUJERES EMBARAZADAS
}

\author{
REINALDO HEREDIA C.* , CLARA INES DE VARGAS**
}

\begin{abstract}
Se estudiaron 198 mujeres en el último trimestre de la gestación con el fin de conocer la frecuencia de la infección por Chlamydia trachomatis, Neisseria gonorrhoeae y Candida albicans diagnosticada empleando cultivos de muestras endocervicales y vaginales. Los porcentajes de infección con los diferentes microorganismos fueron: C. albicans $21 \%$, C. trachomatis $3,5 \%$ y $N$. gonorrhoeae $0,5 \%$. Se destaca la importancia de la investigación de la infección por $C$. trachomatis, en pacientes ginecológicas, en embarazadas, y como parte del estudio preconcepcional.
\end{abstract}

\section{INTRODUCCION}

El diagnóstico temprano de la infección por Chlamydia trachomatis en la mujer embarazada es de gran importancia clínica, ya que ésta puede ser responsable de cervicitis, ruptura prematura de membranas (RPM) y muerte neonatal (1-4). Se ha descrito también que el $50 \%$ de los niños nacidos de madres infectadas con C. trachomatis presentan conjuntivitis y el $20 \%$ neumonia $(1,2,4)$. La tasa de infección por $C$. trachomatis, en las mujeres embarazadas, fluctúa en los diferentes estudios entre el $2 \%$ y el $25 \%(1-3,5)$, siendo 5 a 10 veces mayor que la de la Neisseria gonorrhoeae, este último germen también es de gran patogenicidad en el período de gestación, tanto para la madre como para el feto (6). La incidencia de infección por Candida albicans en mujeres embarazadas puede llegar a ser del $40 \%$, esto se ha atribuido a factores endocrinos y de inmunidad celular. El recién nacido puede infectarse al pasar por el canal del parto y presentar infecciones cutáneas superficiales o en la mucosa oral, así como enfermedades sistémicas (7).

Debido a los efectos adversos de la infección cervicovaginal por estos microorganismos durante la gesta- ción (2-4), y a los escasos datos que se encuentran en nuestra literatura sobre su diagnósstico, especialmente el de infección por $C$. trachomatis (5), quisimos conocer sus porcentajes de infección en una población de mujeres embarazadas.

\section{MATERIALES Y METODOS}

\section{Pacientes}

Se estudiaron 160 mujeres, en el tercer trimestre del embarazo, con leucorrea (sintomáticas) y $38 \mathrm{mu}$ jeres con similares características pero sin flujo patológico (asintomáticas). De cada paciente se consignaron los siguientes datos: semanas de embarazo, edad, paridad, número de abortos, antecedentes de RPM, endometritis, amnionitis, enfermedad pélvica inflamatoria (EPI) e historia de conjuntivitis y/o neumonía en sus hijos recién nacidos.

En el embarazo actual se determinó la presencia de leucorrea, prurito y disuria. Mediante el examen ginecológico se observó si había vulvitis, vaginitis y signos de cervicitis (estimados como erosión y friabilidad), lo mismo que el aspecto de la leucorrea.

* M.D. Jefe Servicio Ginecología y Obstetricia, Caja Nacional de Previsión, Clínica Santa Rosa.

**Bacterióloga, Grupo de Microbiología Instituto Nacional de Salud. 


\section{Toma y Procesamiento de las muestras}

Se tomaron con escobillón tres muestras: una vaginal y dos cervicales (8); la muestra vaginal se empleó para el aislamiento de $C$. albicans (9) y las muestras cervicales para el cultivo de $N$. gonorrhoeae (10) y C. trachomatis (11).

\section{RESULTADOS}

El grupo total de pacientes (sintomáticas y asintomáticas) estuvo conformado por 198 mujeres que tenían entre 35 y 40 semanas de embarazo, con edades comprendidas entre 19 y 39 años. El grupo etário mayor fue el de 19-29 años (78\%). El 70\% de la población había estado en embarazo entre 2 y 5 veces y el $17 \%$ informaron haber tenido abortos. Los antecedentes de endometritis, amnionitis y EPI fueron relatados en menos del $1 \%$ de la población y el de RPM en el $9 \%$. En los antecedentes de los niños se encontró que el $4 \%$ había presentado conjuntivitis y el $2 \%$ neumonía.

Con relación a los síntomas relatados por las 198 pacientes, el $48 \%$ tenía prurito y el $29 \%$ disuria. Al examen ginecológico el $13 \%$ presentó vaginitis y el $6 \%$ vulvitis. En un alto porcentaje de la población se observaron signos de cervicitis: erosión en el $45 \%$ y friabilidad en el $48 \%$. De las 160 mujeres que presentaron leucorrea, el $73 \%$ evidenciaba un flujo blanco grumoso.

En cuanto a los resultados de laboratorio, el germen aislado con mayor frecuencia fue la $C$. albicans que se detectó en el $21 \%$ de los casos. La $C$. trachomatis fue diagnosticada en el 3,5\% aislándose en el 2,4\% $(4 / 168)$ de las sintomáticas y en el $8 \%(3 / 38)$ de las asintomáticas. La $N$. gonorrhoeae se aisló en una sola paciente $(0,5 \%)$.

$\mathrm{Al}$ relacionar los hallazgos de laboratorio con los antecedentes y los síntomas, encontramos lo siguiente: de las pacientes infectadas con C. albicans, el $71 \%$ (27/38) tenían leucorrea, el 66\% (23/35) presentaban prurito, el $44 \%(15 / 34)$ disuria, el 9\% (2/22) vulvitis y ninguna $(0 / 22)$ tuvo vaginitis. La paciente de la cual se aisló $N$. gonorrhoeae tenia erosionado el cervix pero su flujo era blanco grumoso. Las pacientes infectadas con $C$. trachomatis no relataron antecedentes de abortos, amnionitis ni EPI. La RPM y/o la endometritis se habia presentado en el $14 \%$ de los casos (1/7); la conjuntivitis y la neumonía en sus hijos recién nacidos, no fue informada por ninguna de las pacientes. El $33 \%(2 / 6)$ tenían síntomas de disuria y los signos de cervicitis fueron encontrados así: erosión en el $50 \%$ (3/6) y friabilidad en el $40 \%(2 / 5)$.

\section{DISCUSION}

Los resultados anteriores demuestran que, de los tres gérmenes estudiados en la vagina y el cervix, en este grupo de pacientes, el que con mayor frecuencia se aisló fue la $C$. albicans, lo que está de acuerdo con la literatura (12). El aislamiento ocurrió en mayor proporción que en mujeres que acuden a consulta ginecológica general (13); también se confirmó que los síntomas que más se relacionaron con la infección por C. albicans fueron el flujo y el prurito, como ha sido descrito recientemente por Odds y colaboradores y hallado en uno de nuestros trabajos anteriores $(13,14)$.

La baja frecuencia con que se aisló la $N$. gonorrhoeae en este tipo de población, es semejante a lo encontrado por otros grupos (13).

El hallazgo de infección por $C$. trachomatis en el embarazo se encuentra en el rango descrito por otros autores (2-5), incluyendo el único trabajo colombiano en el cual esta infección fue diagnósticada en el 3,3\% del grupo en embarazo estudiado. Sin embargo, estos porcentajes de infección son inferiores a los determinados por nosotros en un grupo de pacientes no embarazadas $(13,15)$, esta falta de correlación en el porcentaje de aislamiento de la $C$. trachomatis en pacientes ginecológicas y obstétricas, puede deberse a las diferencias en clase social y costumbres sexuales de los dos grupos.

El hecho de aislarse la $C$. trachomatis en proporciones semejantes en las pacientes sintomáticas y asintomáticas, señala la importancia de su búsqueda en el exámen ginecológico periódico rutinario, así como en la consulta preconcepcional, logrando con ello prevenir, tanto las anexítis y obstrucciones tubáricas, como las complicaciones que suelen ocurrir por la transmisión vertical de estos microorganismos (2).

Los signos clínicos de cervicitis (erosión y friabilidad) no ayudaron al diagnóstico presuntivo de la infección por $C$. trachomatis, como había sido registrado en un trabajo anterior (13), en el que consideramos 
la friabilidad del endocervix como el signo clínico que permitía una mayor orientación diagnóstica para el ginecólogo. Esto se debe a que la friabilidad es una condición natural en la gestación avanzada (16).

Es importante realizar otros estudios en diferentes tipos de poblaciones colombianas de mujeres embarazadas con el propósito de conocer la verdadera frecuencia de infección por $C$. trachomatis.

\section{SUMMARY}

The frequency of cervical and vaginal infection with Chlamydia trachomatis, Neisseria gonorrhoeae andCandida albicans was studied in 198 pregnant women in their last trimester. The diagnosis was made culturing endocervical and vaginal specimens by standard procedures. The percentages of infection were: $21 \%$ for C. albicans, $3.5 \%$ C. trachomatis and $0.5 \% \mathrm{~N}$. gonorrhoeae. The diagnosis of chamydial infections in gynecologic and pregnant patients is emphasized.

\section{AGRADECIMIENTOS}

A la doctora Martha Urueta, residente de Ginecología de la Clínica San Rafael, por su contribución en la toma de las muestras y a la bióloga Nelly Ordoñez por su excelente colaboración en el procesamiento de las muestras.

\section{BIBLIOGRAFIA}

1. Center For Disease Control. Chlamydia trachomatis infections: policy guidelines for prevention and control. MMWR 1985; 34 (Suppl): 53S-74S.

2. Monif GRG. The impact of Chlamydia trahomatis on mother and infant. In: Impact on the fetus of parental sexually transmitted disease. Ann N Y Acad Sci 1988; 549: 31-38.

3. Alexander ER, Harrison HR. Role of Chlamydia trachomatis in parinatal infection. Rev Infect Dis 1982; 5: 713-719.

4. Harrison HR, English MG, Lee CK, Alexander ER. (bs)Chlamydia trachomatis infant pneumonitis: comparison with matched controls and other infant pneumonitis. N Engl J Med 1978; 298: 702-708.
5. Robledo J, Trujillo LF, Arboleda G, Trujillo H, Mejia GI. Chlamydia trachomatis en síndromes infecciosos de mujeres, en Medellín Colombia, Sur América, Rev Colomb Obstet Ginecol 1987; 38: 175-186.

6. Alexander ER.. Gonorrhea in the newborn. In: Impact on the fetus of parental sexually transmitted diasease. Ann N Y Acad Sci 1988; 549: 180-186.

7. Vulvoviginal Candidiasis: Current Views. Symposium. Am J Obstet Gynecol 1988; 158: 985-996.

8. Isenberg HD, Washington JA, Balows A, Sonnenwirth $\mathrm{AC}$. Collection, handling and processing of specimens. In: EH Lennette, A Balows, WJ Hausler, HJ Shadomy (Eds). Manual of Clinical Microbiology. 4th Ed. American Society for Microbiology. Washington 1985; 73-98.

9. Cooper BH, Silva Hutner M. Yeast of Medical Importance. In: EH Lennette, A Balows, WJ Hausler, HJ Shadomy (Eds). Manual of Clinical Microbiology. 4th Ed. American Society for Microbiology. Washington 1985; 526-541.

10. Guzmán M, Vargas $\mathrm{Cl}$. Neisseria. En Microbiología Médica, Manual de Procedimientos. Serie de Publicaciones Científicas No. 14, Instituto Nacional de Salud, Bogotá, 1988; 127-150.

11. Bird BR, Forrester FT. Laboratory diagnosis of Chlamydia trachomatis infections. U.S. Department of Health and Human Services. Centers for Diasease Control. Atlanta GA. 1981.

12. Paavonen J, Pennti K, et al. Prevalence of nonspecific vaginitis and other cervicovaginal infections during the third trimester of pregnancy. Sex Transm Dis 1986; 13: 5-8.

13. Heredia R, Vargas Cl, Castañeda E. Prevalencia de los agentes etiológicos de la vaginitis en pacientes de consulta ginecológica general. Acta Médica Colombiana. 1990; 15 (2): 92-99.

14. Odds FC, Webster CE, Mayuranathan P, Simmons PD. Candida concentrations in the vagina and their association with sings and symptoms of vaginal candidosis. $\mathrm{J}$ Med Vet Mycol 1988; 26: 277-283.

15. Vargas $\mathbf{C l}$, Heredia R, Castañeda E. Infección por Chlamydia trachomatis en pacientes con neoplasia intracervical. Revista Colombiana de Obstetricia y Ginecología. 1990; 41 (2): 99-105.

16. Pritchard JA, Macdonal PC. Obstetricia de Williams. 2 ed. Salvat, Barcelona, 1979; 174-206. 